\title{
Forces behind the Use of Herbs during Pregnancy by Zimbabwean Women: A Case of Gweru District
}

\author{
Panganai Tsitsi $^{1^{*}(\mathbb{D})}$, Shoko Tabona ${ }^{2}$ (), Mufunda Esther ${ }^{1}$ (i) \\ ${ }^{1}$ Heath Science Department, Zimbabwe Open University, Harare, Zimbabwe \\ ${ }^{2}$ Department of Religious Studies, University of Zimbabwe, Harare, Zimbabwe \\ Email: ^tsitsipanganai@gmail.com, shokotab@yahoo.com, esther.mufunda8@gmail.com
}

How to cite this paper: Tsitsi, P., Tabona, S. and Esther, M. (2021) Forces behind the Use of Herbs during Pregnancy by Zimbabwean Women: A Case of Gweru District. Journal of Biosciences and Medicines, 9, 37-49.

https://doi.org/10.4236/jbm.2021.95005

Received: March 29, 2021

Accepted: May 17, 2021

Published: May 20, 2021

Copyright $\odot 2021$ by author(s) and Scientific Research Publishing Inc. This work is licensed under the Creative Commons Attribution International License (CC BY 4.0).

http://creativecommons.org/licenses/by/4.0/

\begin{abstract}
Purpose: The use of herbal remedies is gradually increasing worldwide and Zimbabwe is not left behind. This study therefore sought to explore the forces behind the use of herbs during pregnancy by Zimbabwean women. Materials and methods: A qualitative approach was chosen using the case study design to evaluate the forces. The study was carried out at three maternity hospitals in Gweru. Thirty (30) women who used herbs during pregnancy were purposefully samples and interviewed using a structured interview schedule. The date was analyzed thematically. Results: It was noted that culture and belief system, previous experience as well as significant people in the woman's life were the forces behind the use of herbs in pregnancy. Conclusion: The study concluded that these forces promoted the indiscriminate use of herbs which are passed from mother to daughter without considering the safety of the herbs to the mother and baby. Therefore, more research is needed to analyze the safety of these herbs to ensure that the mother and baby are safe.
\end{abstract}

\section{Keywords}

Pregnancy, Medicinal Plants, Safety, Delivery

\section{Introduction}

The use of herbs during pregnancy is a big challenge in the health sector in Zimbabwe especially to the midwife who has no knowledge on the effects of the herbs. In the African context, use of herbal remedies is a culturally accepted tradition of a woman and its use is heavily depended on the culture and background of the woman. As a result, herbal remedies continue to play a vital role during the gestation period and in labour as it is influenced by the historical 
practices past [1]. Culture is dynamic and constantly changes, so in this modern society, one would expect women to follow the modern trends of treatment but they still take herbs. There must be other things which are compelling the women to take herbs and this is what this study seeks to explore.

The use of herbal medicines is associated with cultural and personal beliefs [1], as these influence the philosophical views on life and health. Woman's culture and beliefs can be a compelling factor in the use of herbs as its use is viewed as a primary source of healthcare. Traditional Medical Practice views the use of herbs as an integral part of the culture of those communities who use them [2]. Culture controls the behaviour of individuals and even health seeking behaviour. According to the theory of reasoned action [3], the behaviour of a woman is determined by the culture and background of the women, her perceptions as well as the influence of the significant people around her. Thus, the significant people in a woman's life can be a factor promoting the use of herbs in pregnancy. However, traditional practices co-exist with modern practice with most of the women exhibiting biculturalism which encompasses the culture of their grandparents and the culture of other persons surrounding them [4]. As a result, a woman can take or is given some herbal remedy whilst in labour to precipitate the delivery as part of the African tradition but goes to deliver at the hospital.

The use of herbs by the women in labour is congruent with their health beliefs and is part of their transition process to motherhood [5]. Pregnant women believe that herbs enable them to control their childbearing process [5] [6]. The other belief of women which makes them use herbs is the assumption that herbs are safer than conventional drugs [6] [7]. Pregnant women used herbs because they perceived them to be more effective than conventional drugs [8]. It can be argued that the erroneous belief that herbal products are superior to manufactured products makes pregnant women prefer to use herbs [2]. These facts support the claims that, "traditional medicine has always been at the heart of most African people and in particular the Shona people of Zimbabwe" [9]. Therefore, use of herbs can be attributed to the beliefs of the women.

Women use herbs, because they want to ensure that their unborn baby is safe. This was evident in a study in which $62 \%$ of the woman who took herbs during pregnancy in the study said that the herbs gave them fewer side effects than conventional drugs [10], thus reassuring them of the safety of the baby from the teratogenic effects of conventional drugs. This is also backed by the findings that women fear to be subjected to teratogenic effects by conventional drugs [7]. As a result, the woman worries on the safety of her baby can force the woman to choose herbal preparations.

Pregnant women, as consumers of health care, have preferences which will influence their choices to opt for natural therapies as opposed to conventional medicines [2]. In the same vein, women opt for herbs after they have compared experiences between conventional healthcare professionals and complementary medicine practitioners [6]. Dissatisfaction with the attitudes of midwives at health institutions may make the woman to decide on self-medication with her- 
bal preparations at home [7]. As a result, women may decide to use herbs due to dissatisfaction with the results from orthodox pharmaceuticals and the belief that herbal medicines might be effective [2]. In addition, the user friendliness of the herbs was given as the reason for choosing herbs over biomedicine [9].

On the other hand, the choice to use herbs is based on the individual's experience [11]. For the primigravida, who is pregnant for the first time, the experience of the significant others around them is used thus supporting the theory of reasoned action which propounds that an individual's decision is controlled by her close people. The pregnant woman may have a belief or fear that their physician or midwife have not properly identified the problem hence the feeling that herbal remedies are another option [2].

On the other hand, the marketing strategies used by the traditional practitioners makes the herbal preparations to be more alluring to the pregnant mother who wants the best for her baby [2]. The same author goes on to blame the various claims on the efficacy or effectiveness of plant medicines by the traditional practitioners for the increased interest in alternative medicines by pregnant woman. However, the woman's knowledge of herbs and her attitude towards its effectiveness makes her use herbs in pregnancy [6].

The use of herbal preparations in pregnancy is promoted by the high availability of the herbs which are easily accessible without any strict control measures [6]. Herbal preparations are not subjected to quality test and production standards; neither are efficacy nor issues of licensure vigorously controlled before marketing thus making them easily accessible to the pregnant woman without any prescription [12]. In the same vein, the preference of women to use herbs in pregnancy maybe due to the absence of strict regulations like in modern medicines since no prescription is required [11]. It is assumed that herbs are preferred because they are within the vicinity of the users due to mushrooming of self-proclaimed street herbalist [9]. To add to this, the women of today have a high self-esteem which makes them want to move towards self-medication hence herbal remedies with their assumed "safety" tag becomes the target [6]. When the pregnant woman is bombarded with the easily accessible herbs which are highly marketed carrying a safe tag but without any control measures, the temptation to use them is irresistible.

Many women living in rural areas especially in developing countries may decide to use herbs during pregnancy as the herbal preparations from the traditional practitioner may be the main source of health care and sometimes the only source [13]. In their study on the role of Indigenous Medicinal Knowledge in the treatment of illness in Rural Zimbabwe [14] found that women used herbs because of the high prices of the western medicines which they could not afford. In addition to this, it was noted that pregnant women used herbs because they are more accessible without a doctor's prescription [6]. Since these traditional herbs are preferred and are easily accessible, there is need to study them thoroughly so that they are safe for use by these rural folks who at times have no alternative. 
According to the health belief model, Individual perceptions influence how a woman perceives her health [15]. If she perceives that health is important to her and that pregnancy is a condition which can kill her if she does not do something about it, she will be prompted to take action and in this case taking herbs. The same author goes on to explain that modifying factors such as perceived threats and cues to action are factors which are part of the woman's environment that will prompt specific health behaviour in a person. What the woman is told by the elders about pregnancy, labour and the consequences of not taking herbs will be perceived as threats and becomes the cues to action. It can be assumed that if a woman is constantly told that not taking herbs will result in an episiotomy, a long and difficult labour with the possibility of losing her life and that of the baby, she will take the herbs. In addition, if she believes that herbs will prevent such occurrences, then she will take them without any hesitation. The perceived benefits [15] can motivate a woman to take herbs. If a woman perceives the probability of producing a healthy baby after a short labour, she will use the herbs.

The theory of reasoned action (TORA), which is based on the assumption that most behaviours of social relevance are under volitional (wilful) control assumes that individuals are usually quite rational and make systematic use of information available to them [16]. This information which could be from the society, adverts or friends is considered before a decision to engage or not engage in a given behaviour is made [3]. The use of herbal preparations in pregnancy is by choice so the woman, as a person, after obtaining some information, will make a rational decision whether to use or not to use herbal preparations. The theory postulates that an individual's intentions to or not to perform a behaviour is the immediate determinant of that action. The attitude towards the intention is determined by the person's belief that a given outcome will occur if the behaviour is performed [16]. If the pregnant woman believes that herbs will bring a better outcome of her pregnancy, then she will use herbal preparation according to the information gathered. On the other hand, the general subjective norms are all about social pressure put on the individual to perform certain behaviours. This is determined by the person's normative belief about what the important or significant people think should be done and also by the individual's motivation to comply with those other people's wishes or desires. What this means is that, if the important people in the woman's life advise her to take herbs, she will, depending on her motivation to comply with their advice. Whilst a pregnant woman has rights and is not use herbal remedies during pregnancy.

\section{Methods and Materials}

The study was carried out in Gweru urban at the tree hospitals which offer maternity services. Two of the hospitals are private and one is public. This choice enabled the researcher to get the views of the poor who visit the public hospital and the effluent who visit private hospitals. 
A qualitative approach was used as it tends to investigate the meaning that individuals ascribe to social phenomena such as herbal usage in pregnancy [17]. The qualitative method is based on discovery and to understand rather than to predict [17]. The intention of this study is to discover and understand the factors which influence women to use herbs in pregnancy. A case study design was chosen as it provided in-depth study of herbal usage and also provided detailed descriptions of herbal usage in pregnancy. Interviews were done on 30 women who admitted to taking herbs. The sample was chosen using the heterogeneous purposive sampling in order to capture a wide range of conditions in which herbs were taken such as, behaviours, experiences, incidents, or situations [18]. It was anticipated that the women use herbs in various conditions. Some may be forced to take herbs while others use them by choice or certain incidences may have made one to take herbs. In order to make the research rich, the researcher intends to capture different conditions in which herbs are taken. The data was collected using in-depth face to face interviews to allow flexibility and clarification of questions as the interviewer can modify the line of inquiry to enable the interviewee to understand. The interview allowed probing of interesting responses and observation of the non-verbal responses since the information in this study was sensitive and needed observation of body language to probing and bring out the sensitive information [19].

The data was analysed using the constant comparative analysis strategy which allowed data from one interview to be compared it with data from the other interviews noting similarities and differences [20]. The comparisons brought out the aspects of human behaviour and experiences in terms of herbal use which were coded and then analysed thematically according to the research questions. However statistical methods were used in some instances for easy interpretation of data.

The study was carried out in accordance with the Helsinki Declaration Principles. The ethical issues were taken into consideration. Permission to carry out the research was sought from the responsible authorities and approval was granted by the Medical Research Council of Zimbabwe (approval number MRCZ/A2409). The participants consent was obtained through signing the consent form in which matters of confidentiality were explained.

\section{Results}

The study identified three types of forces that make women use herbs as shown in Figure 1.

\subsection{The Woman's Culture and Belief System}

The study found that the woman's culture and beliefs are forces that make women use herbs during pregnancy. Figure 2 indicate the cultural beliefs which lead to herbal use by pregnant women.

Of the women who used herbs, $83.3 \%$ said they did so because it is part of 


\section{factors behind the use of herbs in pregnancy}

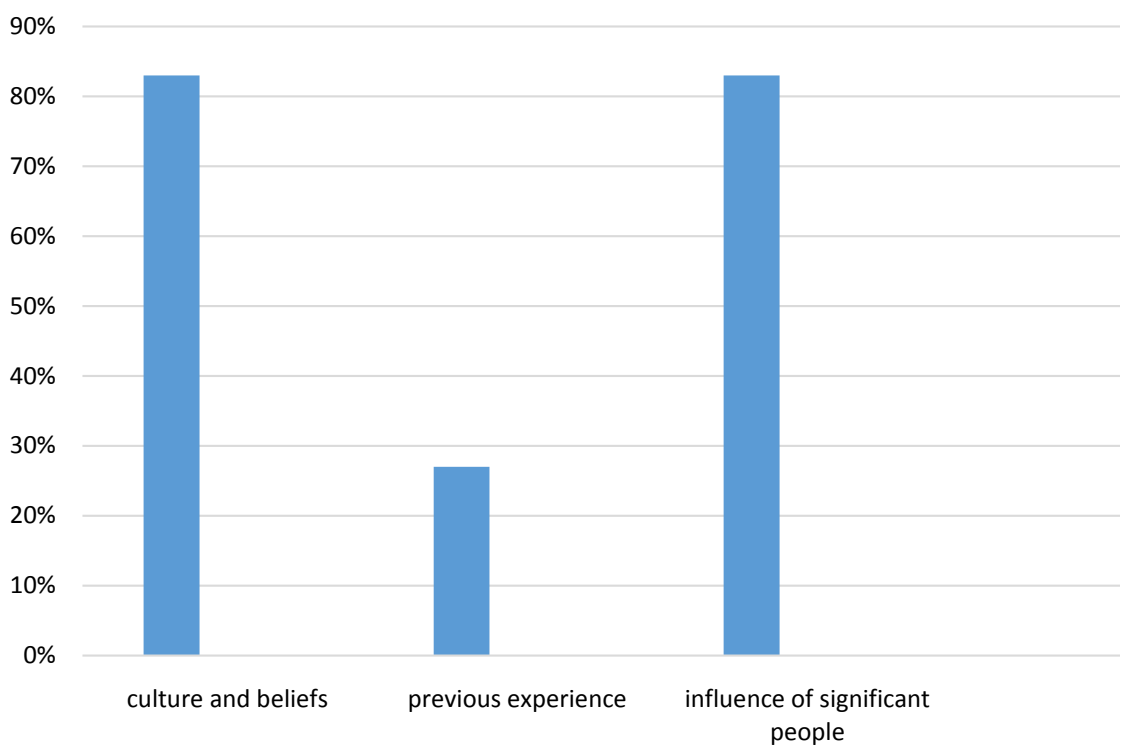

Figure 1. Forces behind use of herbs in labour.

cultural beliefs on use of herbs

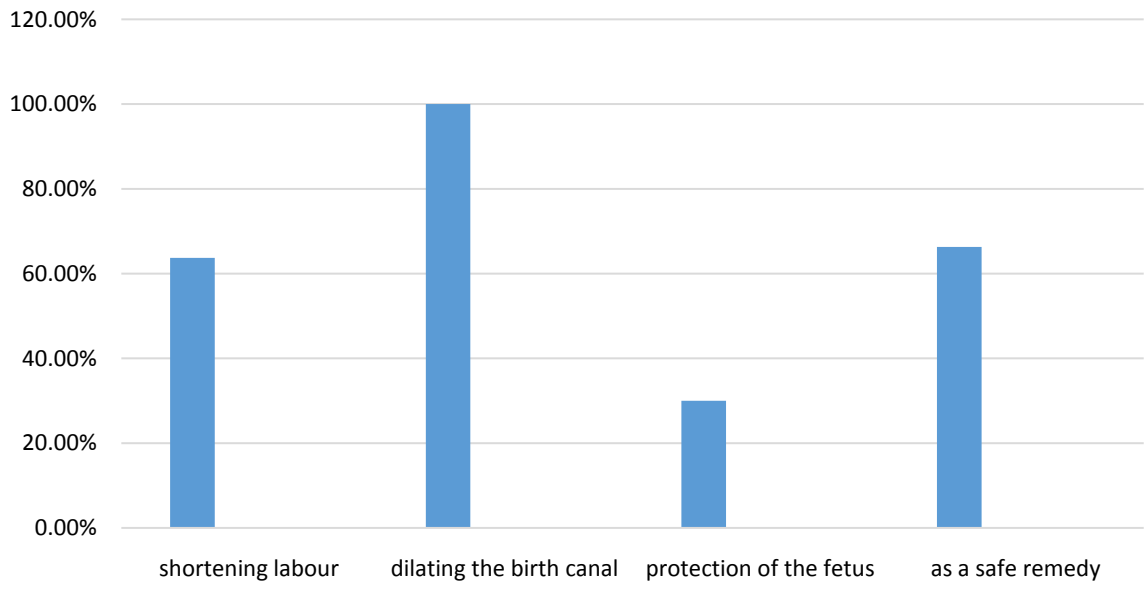

Figure 2. Cultural beliefs behind the use of herbs in pregnancy.

their culture. One of the beliefs that came out of the study was that herbs shorten labour. The majority (63.3\%) of the women who used herbs said that they took herbs to shorten labour. Here are some of the statements from the women who claim to have delivered in a very short time because they had used herbs.

"Labour started at $10.00 \mathrm{hrs}$ and I delivered at $15.00 \mathrm{hrs}$ " (Participant 25)

"My labour was very short lasting about 4 - 5 hours unlike the previous one where I spent a good 3 days in labour" (Participant 15)

"I had a very short labour lasting 3.5 hrs but the pain was terrible" (Participant 29)

"Herbs are effective in that the labour becomes short and easy" (Participant 7)

These statements are indicative of very short labour of about $3-5$ hours. 
Normal active labour takes about 10 - 12 hours excluding the latent stage which varies per individual from hours to days [21].

Some of the participants $(30 \%)$ claimed that they use herbs to protect their unborn baby and to prevent abortions. "I had three abortions so I was given herbs to prevent abortion and now I have my baby" (Participant 3). The women believe that herbs protect their babies from any harm even spiritual as indicated by the quotations from two of the participants;

"I had a still birth so I took some herbs to protect my unborn baby from evil spirits" (Participant 10)

"You can be bewitched so that delivery becomes very difficult so herbs can prevent that" (Participant 1)

This means that the women believed that herbs are important in that they protect the unborn baby.

The study also showed that it is the women's culture and belief that herbs should be taken to prepare the birth canal. All the participants (100\%) claimed that they took herbs for "masuwo" that is widening of the birth canal so that delivery becomes easy and fast. The following statements indicated the use of herbs for "masuwo";

"I was given herbs for 'masuwo' since it is my first child" (Participant 5)

"I was given the herbs so that I will not have stitches" (Participant 16)

"I was given herbs to widen the birth canal and make delivery easy" (Participant 12).

From the findings, it was evident that there was some element of coercion and intimidation so that the young women take the herbs for the intended purpose. One of the participants verbalised this saying, "I was told that if I do not take the 'masuwo' concoction, then I was going to have a very difficult and long labour' (Participant 30). Another participant also said, "My mother in law told me that if anything happens to the baby then I will face the consequences if I do not take the herbs" (Participant 22).

These statements clearly indicate the use of coercion and intimidation as a force for the use of herbs during pregnancy.

The study also showed that the belief that herbs are safe $(66.7 \%)$ is another force behind the use of herbs as indicated by the following interview extracts;

"I have used herbs on all my 4 pregnancies and I did not encounter any problem. After all they are natural and free from toxins" (Participant 29).

"I had no complications and my baby was fine so they are ok with me" (Participant 21).

"I had a C/S on my first baby but now I used herbs and everything was ok" (Participant 7).

"All my children are fine and I delivered them normally because of the herbs I use" (Participant 20).

"I have never had any problems but I use the same herb all the time. Everyone in our family uses them with no problems" (Participant 6).

From the statement above, it can be assumed that women use herbal therapy 
during pregnancy and labour because they believe that the herbs are safe.

\subsection{Herbs Are Used Because of the Previous Experience}

The other force that made women take herbs came out as previous experience. Previous experience can influence women to use herbs especially if the outcome was good as stated by some of the participants;

"I have used herbs on all my 4 pregnancies and I did not encounter any problem. After all they are natural and free from toxins" (Participant 29).

"I had no complications from herbs on all my deliveries so they are ok with me" (Participant 3).

"In all my deliveries, I have used herbs and I delivered normally with no problems so herbs work" (Participant 4).

"I have never had any problems but I use the same herb all the time. Everyone in our family uses them with no problems" (Participant 22).

From the findings, it is quite clear that the participants used herbs repeatedly as verified by the interview extracts above. However, others still used herbs even when the outcome was bad, "Herbs are safe. My child died due to the delayed operation when my child was in distress. The culprits are the midwives not the herbs because all my sisters used the same herb with no problems" (Participant 11). One participant believed that herbs work since her previous experience without herbs was not favourable, "I had a caesarean section on my first baby but now I used herbs and everything was ok so the herbs work" (Participant (26).

\subsection{Herbs Can Be Used Because of the Influence of the Significant People in the Women's Life}

The study results revealed that the significant people in the woman's life can be forces behind the use of herbs in pregnancy. These included the mother, grandmother, aunt, friend and the traditional Birth Attendances (TBAs). The study also identified the sources of herbs as close associates of the pregnant women. The mother was identified as the main source of herbs as the majority, 25 (85\%) of the participants were given herbs by their mothers. The remaining 5 (15\%) were given by a grandmother/aunt or a friend. A mother is the most trusted person by any individual and more so if one is pregnant. All the herbs however, were provided by significant persons of the women and those who had no knowledge on herbs out sourced them. This was explained by one participant who said, "my mother gave me the herbs but she sourced them from the TBA".

\section{Discussion}

The data elicited from the women who used herbs showed that the use of herbs is part of the woman's culture. Of the women who used herbs, $83.3 \%$ said they did so because it is part of their culture. Cultures share a belief that during pregnancy, the mother and the foetus are vulnerable as a result, herbal remedies is heavily depended on the culture and background of the woman and will contin- 
ue to play a pivotal role during the gestation period and in labour just like they did in the past [1]. In addition, pregnant women believe that herbs are able to control their childbearing process [3]. Therefore, culture and beliefs are a force in the use of herbs by women.

The majority (63.3\%) of the women who used herbs said that they took herbs to shorten labour.

The purpose of taking herbs as stated by the participants was to have an easy and fast delivery, so to these women, herbs are effective. Labour lasting about 3 hours is definitely precipitate labour. This is in line with the findings that a woman is given herbs to precipitate delivery as part of the African culture [1]. This belief that herbs make labour quick and easy is a force that propels women to use herbs for who does not what labour to be easy and quick. Accordingly [13], a woman can use her rights as a bio-psycho -social and cultural being to act on her health. This means that if a woman wants a short labour and has the means to obtain one, then she will do so even if it means using herbal preparations. As a result, the need to have a short and easy labour is a force that makes women use herbs.

Some of the participants claimed that they use herbs to protect their unborn baby and to prevent abortions. The African tradition believes in witchcraft and evil spirits of which these things can be dealt with through use of traditional herbs as one of the participant said. These sentiments show that herbs play a significant role in the psycho-spiritual realm of an individual. If a mother perceives that her unborn baby is threatened, then she will take every possible action to prevent that including herbs. This is in accordance with the Health Belief Model (HBM) which looks at perceived threat and perceived benefits as cues to action which is happening with these women. The women perceive a threat to their unborn baby and believe that herbs will protect the baby hence their use during pregnancy. These findings are similar to the claims that the influence of religion and the spiritual consciousness of the pregnant women make them more inclined to use treatment based on their faith rather than scientific beliefs [2].

The study showed that preparation of the birth canal is very important to the expecting mother. However, it appears as if the mothers were being given and not taking herbs. It looks like coercion is being used with some bit of intimidation (...since it is my first child and ... will not have stiches). Every woman is excited about her first child so by stressing on this, the woman will definitely take herbs to have a good outcome of her "first" baby. In the same vein no woman wants stiches on her perineum. So this gentle persuasion and intimidation can be a force to use of herbs in pregnancy. These women took herbs because someone was deciding for them as herbs could be taken due to social pressure from significant people in the pregnant woman's life [1]. In addition, the behaviour of a pregnant woman is influenced by her customs [4]. So in the African custom where elders are respected, it becomes very difficult for the pregnant woman to resist advice and or pressure from her family especially the mother and aunts. 
Fear of a difficult and long labour or episiotomy was being instilled in the mothers so that they take the herbs.

All $30(100 \%)$ of the participants believed that herbal therapy is safe. The belief that herbs are safe can be a force behind their use. These finding concur with the claims that herbal preparations are safe with rare incidences of adverse effects of the mother and baby [14]. Women used herbs because they think that herbs are safer than conventional methods [4]. It is an assumption of the women that because herbs are natural they are safe from toxic effects. If they thought that herbs can be dangerous they would not use them as one of the forces for using herbs was to protect their unborn babies and not to harm them. Those who believe that the herbs are safe had a pleasant. Experience and outcome and these are likely to use them again. This is supported by the argument that the choice to use herbs is based on the individual's previous experience [7].

Previous experience can influence women to use herbs especially if the outcome was good. What this seems to imply is that if the woman used herbs and it worked, she will continue to use them. Even those who had complications blamed the health system as they believed that herbs are safe. Women believe that the outcome of their pregnancy was better off when they use herbs [19] and they also analyse the attitude of the health workers. Some women from the study blamed the health workers for the complications they encountered rather than the herbs. This supports other research finding that the use of herbs is based on individual experiences [9]. Women are comfortable with traditional medicines and are satisfied with the results hence they choose their traditional medicines irrespective of the existence of western medicine [21]. It then can be assumed that the use of herbs can be attributed to previous experience.

The majority 25 (85\%), of the participants were given herbs by their mothers who is a significant person in the pregnant woman's life as claimed by the theory of reasoned action. It becomes difficult for the woman to refuse the herbs being given by her mother especially if it coupled with coercion and threats. So besides preparing the birth canal and fear of a difficult labour, the other force compelling women to take herbs is trust and respect for the elders.

A mother is the most trusted person by any individual and more so if one is pregnant. The Shona culture has a tradition of sending a woman who is pregnant for the first time to her mother's home until she delivers. The mother is then expected to take care of her daughter throughout the last trimester of pregnancy, ensure a safe delivery and teaches her how to care for the infant. So to ensure a safe delivery, the mother gives her daughter herbs which she was given by her mother. This way the herbs are passed from generation to generation and that the family and friends are the sources of herbs [9]. If the mother is not knowledgeable about herbs, she out sources them from the TBAs so as to fulfill her societal expectations and duty. This concurs with the claim that birth attendances are a source of herbal information for pregnant mothers [1]. On the other hand, the marketing strategies used by the traditional practitioners makes the 
herbal preparations to be more alluring to the pregnant mother who wants the best for her baby [14]. The problem however arises as family members who mostly recommended the use of herbs may not have sufficient knowledge to advise pregnant women about the use of herbal drugs [20]. Since the family is the key to herbal usage this becomes another force to herbal use in pregnancy.

\section{Conclusion}

A pregnant woman is capable of making decision pertaining to her pregnancy, but the study revealed that there are a number of forces that compel her to make a decision to take herbs. The African culture places the young mother in a compromised position where she has to take herbs whether she likes it or not. These traditions which are passed from mother to daughter make the mother give her daughter herbs simply, because that's the family tradition. The gentle pressure and intimidation makes the woman take the herbs, because they want the best outcome of pregnancy. The woman's beliefs that herbs are safer and shorten labour coupled with previous experience drive the women to use herbs. There is however the question of the safety of the herbs. The issue of safety of the herbs used during pregnancy needs to be taken seriously. There is need to carry out more studies on the effects of these herbs on the health of the mother and child.

\section{Recommendations}

After analyzing the findings of the study, it is recommended that more research be carried out on the use of African herbs during pregnancy and labour to ensure safe motherhood.

\section{Acknowledgements}

The authors would like to thank the hospital authorities and the participants for allowing this research to be conducted.

\section{Sources of Finance}

There was no funding for this article.

\section{Authorship Contribution}

Panganai Tsitsi: data collection analysis and discussion.

Professor Shoko Tabona: methodology and editing.

Dr Mufunda Esther: introduction and editing.

\section{Conflicts of Interest}

There is no conflict of interest.

\section{References}

[1] WHO Traditional Medicine Strategy 2014-2023; 2015 Geneva.

[2] Ekor, M. (2014) The Growing Use of Herbal Medicines: Issues Relating to Adverse 
Reactions and Challenges in Monitoring Safety. Frontiers in Pharmacology, 4, 177. https://doi.org/10.3389/fphar.2013.00177

[3] Ajzen, I. and Fishbein, M. (1988) Theory of Reasoned Action-Theory of Planned Behavior. University of South Florida, Tampa.

[4] Helman, C. (2007) Culture, Health and Illness. 5th Edition, CRC Press, London. https://doi.org/10.1201/b13281

[5] Hall, H.G., Griffiths, D.L. and McKenna, L.G. (2011) The Use of Complementary and Alternative Medicine by Pregnant Women: A Literature Review. Midwifery, 27, 817-824. https://doi.org/10.1016/j.midw.2010.08.007

[6] Peprah, P., Agyemang-Duah, W., Arthur-Holmes, F., Budu, H.I., Abalo, E.M., Okwei, R. and Nyonyo, J. (2019) "We Are Nothing without Herbs": A Story of Herbal Remedies Use during Pregnancy in Rural Ghana. BMC Complementary and Alternative Medicine, 19, Article No. 65. https://doi.org/10.1186/s12906-019-2476-x

[7] Pallivalapila, A., R, Stewart, D., Shetty, A., Pande, B., Singh, R. and McLay, J.S. (2015) Use of Complementary and Alternative Medicines during the Third Trimester. Obstetrics \& Gynecology, 125, 204-211. https://doi.org/10.1097/AOG.0000000000000596

[8] Cuzzolin, L., Zaffani, S., Murgia, V., Gangemi, M., Meneghelli, G., Chiamenti, G.P. and Benoni, G. (2003) Patterns and Perceptions of Complementary/Alternative Medicine among Paediatricians and Patients' Mothers: A Review of the Literature. European Journal of Pediatrics, 162, 820-827. https://doi.org/10.1007/s00431-003-1313-9

[9] Shoko, T. (2018) Traditional Herbal Medicine and Healing in Zimbabwe. Journal of Traditional Medicine \& Clinical Naturopathy, 7, 254. https://doi.org/10.4172/2573-4555.1000254

[10] Nordeng, H. and Havnen, G.C. (2005) Impact of Socio-Demographic Factors, Knowledge and Attitude on the Use of Herbal Drugs in Pregnancy. Acta Obstetricia et Gynecologica Scandinavica, 84, 26-33. https://doi.org/10.1111/j.0001-6349.2005.00648.x

[11] John, L.J. and Shantakumari, N. (2015) Herbal Medicines Use during Pregnancy: A Review from the Middle East. Oman Medical Journal, 30, 229. https://doi.org/10.5001/omj.2015.48

[12] Kasilo, O.M.J. and Trapsida, J.M. (2011) Decade of African Traditional Medicine, 2001-2010. Afr. Health Mon. (Special Issue), 14, 25-31.

[13] World Health Organization (2015) Traditional Medicine. Macao. http://www.who.int/medicines/areas/traditional/en/

[14] Percyslage, C., Robert, M. and Feddious, M. (2007) The Role of Indigenous Medicinal Knowledge (IMK) in the Treatment of Ailments in Rural Zimbabwe: The Case of Mutirikwi Communal Lands. Journal of Sustainable Development in Africa, 9 , 26-43.

[15] Rosenstock, I.M. (1974) Historical Origins of the Health Belief Model. Health Education \& Behavior, 2, 328-335. https://doi.org/10.1177/109019817400200403

[16] Ajzen, I. and Fishbein, M. (2005) The Influence of Attitudes on Behaviour. In: Albarracín, D., Johnson, B.T. and Zanna, M.P., Eds., The Handbook of Attitudes, Lawrence Erlbaum Associates, Mahwah, 173-221.

[17] Stausberg, M. and Engler, S. (2013) The Routledge Handbook of Research Methods in the Study of Religion. Routledge, London.

https://doi.org/10.4324/9780203154281 
[18] Onwuegbuzie, A.J. and Collins, K.M. (2007) TA Typology of Mixed Methods Sampling Designs in Social Science Research. The Qualitative Report, 12, 281-316.

[19] Polit, D.F. and Hungler, B.P. (2013) Essentials of Nursing Research: Methods, Appraisal and Utilisation. 8th Edition, Lippincott Williams \& Wilkins, Philadelphia.

[20] Thorne, S. (2000) Data Analysis in Qualitative Research. Evidence-Based Nursing, 3, 68-70. https://doi.org/10.1136/ebn.3.3.68

[21] Shin, H.-K., Jeong, S.-J., Lee, M.S. and Ernst, E. (2013) Adverse Events Attributed to Traditional Korean Medical Practices: 1999-2010. World Health Organization Bulletin, 91, 569-575. https://doi.org/10.2471/BLT.12.111609 